\title{
Impact of Blackbody Warm-Up Cool-Down Cycle on the Calibration of Aqua MODIS and S-NPP VIIRS Thermal Emissive Bands
}

\author{
Yonghong $\mathrm{Li}^{\circledR}$, Member, IEEE, Xiaoxiong Xiong, Jeff McIntire, and Aisheng $\mathrm{Wu}^{\circledR}$
}

\begin{abstract}
This paper evaluates the calibration quality during the blackbody (BB) warm-up cool-down cycle for thermal emissive bands onboard Aqua Moderate Resolution Imaging Spectroradiometer (MODIS) and Suomi National Polar-orbiting Partnership (S-NPP) Visible Infrared Imaging Radiometer Suite (VIIRS). This evaluation utilizes data from Aqua MODIS Collection 6 Level-1B products and VIIRS Sensor Data Records in 6-min granule format provided by the NASA Land Science Investigator-led Processing System. Nearly simultaneous hyperspectral measurements from the Aqua Atmospheric Infrared Sounder (AIRS) and the S-NPP Cross-track Infrared Sounder (CrIS) are used as references for MODIS and VIIRS, respectively. Each AIRS footprint of $13.5 \mathrm{~km}$ is co-located with multiple MODIS pixels while each CrIS field of view of $14 \mathbf{~ k m}$ is co-located with multiple VIIRS pixels. The corresponding AIRSsimulated MODIS and CrIS-simulated VIIRS radiances are derived by convolutions based on sensor-dependent relative spectral response functions. In this paper, the analysis mainly focuses on the bands that are used in sea surface temperature products. The results show that there is virtually no impact for MODIS bands 22 and 23 and bands 31 and 32 for a BB temperature below $290 \mathrm{~K}$; however, when the BB temperature increases above $290 \mathrm{~K}$, the impact is up to $0.3 \mathrm{~K}$ for bands 22 and 23 and $0.05 \mathrm{~K}$ for bands 31 and 32, respectively. For VIIRS, BB temperaturedependent drifts are observed in M15 and M16, which can reach 0.15 and $0.1 \mathrm{~K}$, respectively, over the operational $\mathrm{BB}$ temperature range and the VIIRS brightness temperature range.
\end{abstract}

Index Terms-Aqua Atmospheric Infrared Sounder (AIRS), blackbody (BB), calibration, Cross-track Infrared Sounder (CrIS), Moderate Resolution Imaging Spectroradiometer (MODIS), thermal emissive bands (TEBs), Visible Infrared Imaging Radiometer Suite (VIIRS).

\section{INTRODUCTION}

$\mathbf{S}$ ATELLITE missions for earth observations have collected long-term science data for better understanding of the earth's system. Various sensors collect data with different spatial resolutions, ranging from low resolution (e.g., $4 \mathrm{~km}$ for AVHRR global area coverage data) to high resolution (e.g., $0.41 \mathrm{~m}$ from the commercial satellite GeoEye1). Their spectral coverage is achieved either through separate spectral channels

Manuscript received June 28, 2017; revised October 11, 2017 and November 20, 2017; accepted November 22, 2017. Date of publication December 25, 2017; date of current version March 23, 2018. (Corresponding author: Yonghong $\mathrm{Li}$.)

Y. Li, J. McIntire, and A. Wu are with the Science Systems and Applications, Inc., Lanham, MD 20706 USA (e-mail: Yonghong.Li@ssaihq.com).

$\mathrm{X}$. Xiong is with the Sciences and Exploration Directorate, Goddard Space Flight Center, National Aeronautics and Space Administration, Greenbelt, MD 20771 USA.

Color versions of one or more of the figures in this paper are available online at http://ieeexplore.iee.org.

Digital Object Identifier 10.1109/TGRS.2017.2779105 or through interferometers, spanning wavelengths from visible to thermal infrared. These satellite data have been used in environmental management (land use assessment, geology, forestry, etc.) as well as the investigations of climate change, the atmosphere, and natural hazards. The calibration of earth observation data is crucial to ensure the quality of the collected data for scientific studies.

The Moderate Resolution Imaging Spectroradiometer (MODIS), a key instrument on National Aeronautics and Space Administration (NASA)'s Earth Observation System missions, is aboard the Terra and Aqua satellites and has operated for over 17 and 15 years, respectively. The Visible Infrared Imaging Radiometer Suite (VIIRS) instrument is designed to extend the measurements from MODIS [1], [2]. It has operated on-orbit for over five years as a major earth observing sensor aboard the Suomi National Polar-orbiting Partnership (S-NPP) satellite. Both instruments have thermal emissive bands (TEBs) covering a spectral range from 3.6 to $14.4 \mu \mathrm{m}$ for MODIS (bands 20-25 and 27-36) and from 3.6 to $12.5 \mu \mathrm{m}$ for VIIRS (imaging bands I4 and I5 and moderate-resolution bands M12-M16). These thermal infrared remote sensing bands have been used successfully in the applications of surface temperature mapping, cloud mask, etc. [3]-[7].

The blackbody (BB) is an onboard calibrator for the TEBs on both MODIS and VIIRS. The BB is temperature controlled and its temperature is monitored by embedded thermistors, 12 on MODIS and 6 on VIIRS. To monitor or estimate the TEB calibration coefficients, periodically scheduled BB warm-up and cool-down (hereafter WUCD) cycles are implemented to acquire the detector response over a range of BB temperatures from instrument ambient (around $270 \mathrm{~K}$ for MODIS and $267 \mathrm{~K}$ for VIIRS) to $315 \mathrm{~K}$. The TEB performance of MODIS and VIIRS is monitored and evaluated based on the stability of both the on-orbit BB calibration data and their earth view Level-1B (L1B) products [8]-[13]. The intercomparison with different sensors is another independent approach to track sensor on-orbit performance. This is usually conducted using simultaneous nadir observation (SNO) measurements [13]. Near-surface temperature observations can also be used to track the long-term calibration stability of the TEBs [14]. Overall, the TEB performance of Aqua MODIS (hereafter MODIS) and S-NPP VIIRS (hereafter VIIRS) has been stable since launch [9], [15]. During the WUCD cycle, it is expected that the current calibration algorithms should capture the changes in the thermal environment and produce a consistent result from the nominal scan-by-scan calibration. 
However, some issues still exist. For example, the Aqua MODIS bands 33, 35, and 36 saturate at BB temperatures above approximately 293, 296, and $301 \mathrm{~K}$, respectively, which need to be calibrated separately [16]. For VIIRS, up to $0.3-\mathrm{K}$ spikes in sea surface temperature (SST) have been observed during the quarterly WUCD cycle. As a result, a new calibration algorithm was proposed to address the issue [17].

This paper will evaluate the calibrated TEB data during WUCD cycles for MODIS and VIIRS. The focus is on the impact of the BB temperature on MODIS and VIIRS TEB calibration for the bands used in SST products, MODIS bands 22 and 23 (wavelengths of 3.96 and $4.05 \mu \mathrm{m}$ for the short-wave SST algorithm), and 31 and 32 (wavelengths of 11 and $12 \mu \mathrm{m}$ for the long-wave SST algorithm), and VIIRS bands M15 and M16 (wavelengths of 11 and $12 \mu \mathrm{m}$ for the long-wave SST algorithm) [4], [6]. We also report the results of VIIRS bands I5 and M13 since they are in the SST spectral range. The results shown in this paper will provide helpful information on the explanation of the spikes observed in VIIRS SST products and its comparison with MODIS. The SNO data from the Atmospheric Infrared Sounder (AIRS) and the Cross-track Infrared Sounder (CrIS) are used as references since they are also onboard the Aqua and S-NPP satellites, respectively. Both AIRS and CrIS are hyperspectral infrared sounders. AIRS is a grating spectrometer with multiple detector arrays for the corresponding spectral channels while CrIS is an interferometer. Details on their calibration and performance can be found in [18]-[20].

Section II reviews the algorithms for MODIS and VIIRS TEB on-orbit calibrations. Section III provides some relevant background on the four instruments, AIRS, MODIS, CrIS, and VIIRS, as well as the AIRS-MODIS and CrIS-VIIRS SNO intercomparison methodology. Results of the WUCD impact on MODIS and VIIRS TEBs will be shown in Section IV, including data collection, data analysis, intercomparison results, and discussions. A summary of our analysis is contained in Section V.

\section{MODIS AND VIIRS TEB ON-ORBIT CALIBRATION}

The $\mathrm{BB}$ assembly is designed to provide known radiance levels for the TEB calibration with a close-to-unity emissivity (greater than 0.987 for MODIS and 0.996 for VIIRS) and an excellent temperature uniformity [2], [21], [22]. The onboard $\mathrm{BB}$ and space view (SV) observations are used to perform the onboard calibrations for TEBs in both the MODIS and VIIRS instruments. The temperatures of the onboard BB are controlled (285 K on MODIS and 292.5 K on VIIRS) during normal operations. The TEB calibration converts the digital response of a detector to the sensor's at-aperture radiance. A second-order polynomial model is currently used in VIIRS and MODIS TEB calibrations with different approaches to estimate the coefficients.

\section{A. Aqua MODIS TEB Calibration}

The MODIS TEB radiance is calculated by

$$
\begin{aligned}
L_{\mathrm{EV}}=\frac{1}{\mathrm{RVS}_{\mathrm{EV}}}\left(a_{0}+b_{1} \cdot d n_{\mathrm{EV}}+a_{2} \cdot d n_{\mathrm{EV}}^{2}\right. & \\
& \left.-\left(\mathrm{RVS}_{\mathrm{SV}}-\mathrm{RVS}_{\mathrm{EV}}\right) \cdot L\left(T_{\mathrm{SM}}\right)\right)
\end{aligned}
$$

where $L, d n$, RVS, and $T$ represent radiance, the background corrected digital counts, relative response versus scan angle, and temperature, respectively, EV and SM refer to the earth view and scan mirror side, and $a_{0}, b_{1}$, and $a_{2}$ are the calibration coefficients which depend on band, detector, and mirror side. The offset $\left(a_{0}\right)$ and nonlinear coefficient $\left(a_{2}\right)$ are estimated from WUCD data while $b_{1}$ is determined by viewing $\mathrm{BB}$ and SV on a scan-by-scan basis with the exception of band 21, which is derived from BB cool-down (CD) data. In the current Aqua MODIS Collection 6 calibration algorithm, bands 31 and 32 use a free fit $a_{2}$ (with $a_{0}$ set to 0 ) from the detectors' responses to the $\mathrm{BB}$ source during the $\mathrm{CD}$ portion of its WUCD events since there was a gain change right before the launch for Aqua. For the other TEBs, the coefficients $a_{0}$ and $a_{2}$ are based on prelaunch testing and are adjusted with an iterative approach to account for any on-orbit drift [23].

\section{B. S-NPP VIIRS TEB Calibration}

For S-NPP VIIRS, a quadratic algorithm is currently used in the TEB calibration, which is expressed by [24]

$$
\begin{array}{r}
L_{\mathrm{EV}}=\frac{1}{\mathrm{RVS}_{\mathrm{EV}}}\left[F \cdot \sum_{i=0}^{2} c_{i} \cdot d n_{\mathrm{EV}}^{i}-\left(\mathrm{RVS}_{\mathrm{EV}}-\mathrm{RVS}_{\mathrm{SV}}\right)\right. \\
\left.\cdot \frac{\left(1-\rho_{\mathrm{rta}}\right) \cdot L\left(T_{\mathrm{rta}}\right)-L\left(T_{\mathrm{ham}}\right)}{\rho_{\mathrm{rta}}}\right]
\end{array}
$$

where rta and ham represent rotating telescope assembly (RTA) and half-angle mirror (HAM), respectively, $\rho$ is the spectral reflectance of the RTA, $c_{i}(i=0,1,2)$ are temperature-dependent calibration coefficients for each band, detector, and HAM side, and $F$ is a calibration scaling factor applied to the estimated radiance represented by the quadratic polynomial model. Currently, $c_{i}$ are determined using prelaunch coefficients interpolated to on-orbit temperatures. The scaling factor $F$ is calculated on a scan-by-scan basis from on-orbit BB measurements. Results from VIIRS BB WUCD data analysis are closely monitored for potential updates to the coefficients if necessary.

\section{BB WUCD Operations for MODIS and VIIRS}

During an Aqua MODIS WUCD cycle, the BB temperature is first allowed to $\mathrm{CD}$ from its operational temperature $(285 \mathrm{~K}$ ) to $270 \mathrm{~K}$, which requires about $18 \mathrm{~h}$. Then, a series of commands is sent out to set the BB temperature to 280, 285, 290 , and $315 \mathrm{~K}$. Shortly after the BB temperature reaches $315 \mathrm{~K}$, a command is sent to turn OFF the BB heater and the $\mathrm{BB}$ temperature starts a full range $\mathrm{CD}$ cycle to approximately $270 \mathrm{~K}$, which takes about $20 \mathrm{~h}$. Then, the BB is heated until its temperature reaches its operation temperature $(285 \mathrm{~K})$ and the WUCD cycle is finished. A whole WUCD cycle usually takes $53 \mathrm{~h}$. A typical BB temperature profile during a WUCD cycle is illustrated in Fig. 1(a).

The current VIIRS WUCD cycle is different from MODIS WUCD cycle. During a VIIRS WUCD event, the BB is heated first from its operational temperature $(292.5 \mathrm{~K})$ to $315 \mathrm{~K}$ by sending commands to set the BB temperature to 297.5, 302.5, $307.5,312.5$, and $315 \mathrm{~K}$ with a 3 -h interval for each step. 


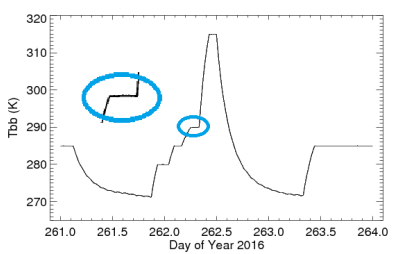

(a)

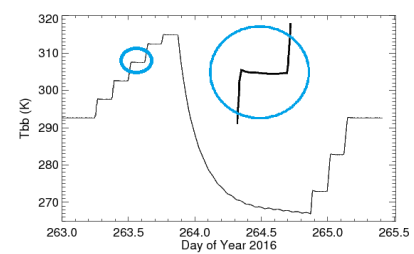

(b)
Fig. 1. BB temperature change during WUCD cycles for (a) MODIS 2016/261 and (b) VIIIRS 2016/263.

After the $\mathrm{BB}$ temperature reaches $315 \mathrm{~K}$, the $\mathrm{BB}$ heater is turned OFF and the BB cools down for $24 \mathrm{~h}$. The BB heater is then turned $\mathrm{ON}$ and the $\mathrm{BB}$ temperature recovers to its nominal temperature $292.5 \mathrm{~K}$ pausing for two settings (272.5 and $282.5 \mathrm{~K}$ ) along the way. The whole WUCD cycle takes $\sim 46 \mathrm{~h}$ to complete, which is $7 \mathrm{~h}$ less than that taken by Aqua MODIS. Fig. 1(b) shows the BB temperature change for VIIRS during 2016/263 WUCD event.

Comparing the $\mathrm{BB}$ temperature changes during the WUCD period, it is observed that the MODIS BB temperature increased relatively smoothly to the setting temperature level whereas the VIIRS BB temperature reaches beyond the setting temperature level first and then falls back to the level (see the zoomed-in portions of Fig. 1).

\section{SNO DATA COLlection AND PROCESSING}

\section{A. MODIS and AIRS Data}

MODIS has 16 TEBs (3.6-14.4 $\mu \mathrm{m})$ with 1-km ground resolution at nadir. Each TEB has ten detectors aligned along the track direction. Its earth view scenes contain 1354 samples in the scan direction covering a scan-angle range of $\pm 55^{\circ}$ off nadir. On the same Aqua satellite, AIRS's thermal infrared spectra contains 2378 spectral channels, spanning a range from 3.7 to $15.4 \mu \mathrm{m}$. AIRS is an across track scanning system centered at nadir. A nominal scan line covers 90 infrared footprints within a scan range of $\pm 49.5^{\circ}$, which corresponds to a ground resolution of $13.5 \mathrm{~km}$ at nadir for the Aqua satellite's altitude of $705.3 \mathrm{~km}$.

\section{B. VIIRS and CrIS Data}

VIIRS has seven TEBs, two image-resolution bands (I-bands, $375 \mathrm{~m}$ at nadir) I4 and I5 and five moderateresolution bands (M-bands, $750 \mathrm{~m}$ at nadir) M12-M16. There are 32 detectors in an I-band and 16 detectors in an M-band aligned along the track direction. VIIRS TEBs cover a spectral range from 3.6 to $12.5 \mu \mathrm{m}$. VIIRS earth view scenes of the moderate-resolution bands have 3200 samples in the scan direction covering a scan-angle range of $\pm 56^{\circ}$ off nadir. The same S-NPP satellite also carries the CrIS instrument, a Fourier transform spectrometer, measuring radiance for retrieving profiles of temperature, pressure, moisture, etc. It has 1305 spectral channels covering three wavelength ranges: short-wave infrared (3.92-4.64 $\mu \mathrm{m})$, mid-wave infrared (5.71-8.26 $\mu \mathrm{m})$, and long-wave infrared (9.14-15.38 $\mu \mathrm{m})$. There are 30 earth-scene views (referred to as fields of regard) in a scan covering $\pm 50^{\circ}$. Each view
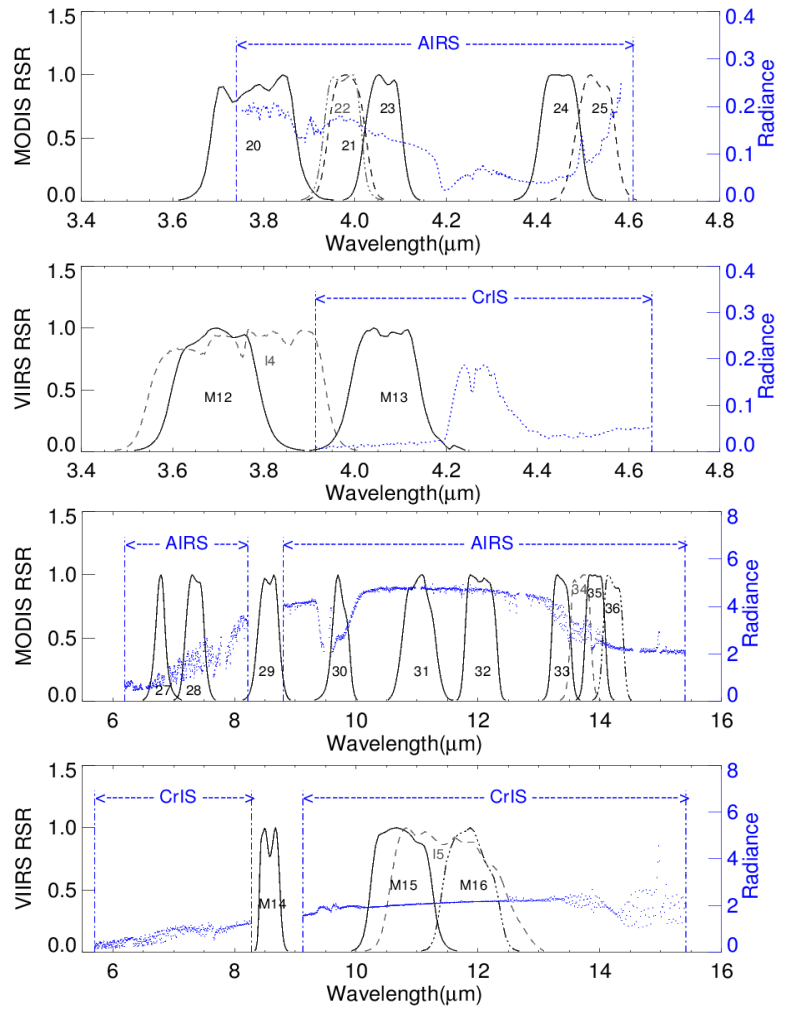

Fig. 2. MODIS and VIIRS spectral distribution as well as AIRS and CrIS spectral coverage (radiance unit: $\mathrm{W} / \mathrm{m}^{2} / \mu \mathrm{m} / \mathrm{sr}$ ).

position contains a $3 \times 3$ field-of-view (FOV) array. The spatial resolution of each FOV is $14 \mathrm{~km}$ at nadir.

\section{SNO Data Processing for Evaluations}

Since MODIS and AIRS are onboard the Aqua satellite and VIIRS and CrIS are onboard the S-NPP satellite, frequent SNO data can be collected to compare the measurements from the two pairs of sensors. In our analysis, all SNO data need to be converted to the same grid, both spectrally and spatially. The hyperspectral AIRS (or CrIS) data are integrated to match each MODIS (or VIIRS) spectral band while the high spatial resolutions of MODIS (or VIIRS) data are aggregated into each AIRS (or CrIS) FOV in order to match the spatial footprint.

1) Spectral Simulation: The sensor-dependent relative spectral response (RSR) describes the system transmission as well as the detector sensitivity. Fig. 2 illustrates the spectral profiles of MODIS and VIIRS TEBs as well as spectral coverage of AIRS and CrIS. As shown in Fig. 2, all MODIS TEB wavelengths are within the AIRS hyperspectral coverage, except for bands 20 and 29. CrIS spectra fully covers VIIRS TEBs I5, M15 and M16, and $99.9 \%$ of band M13.

To compare the radiance measurements from MODIS and AIRS, the RSR function of each MODIS band needs to be applied to each of AIRS channels using interpolation, then the simulated radiances from the AIRS measurements are calculated for each MODIS band

$$
L_{\text {simulated }}=\frac{\int_{\lambda_{1}}^{\lambda_{2}} L_{\mathrm{hi}}(\lambda) \cdot \operatorname{RSR}(\lambda) \cdot d \lambda}{\int_{\lambda_{1}}^{\lambda_{2}} \operatorname{RSR}(\lambda) \cdot d \lambda}
$$


where " $L_{\mathrm{hi}}$ " is the band-dependent interpolated radiance from AIRS measurements. Linear interpolation is used in this analysis. $\left[\lambda_{1}, \lambda_{2}\right]$ is the wavelength range of a band. The same method is used to compare between VIIRS and CrIS.

2) Spatial Aggregation: In this SNO data processing, AIRS and CrIS data are taken from six footprints and two center field of regards $\left(\sim \pm 3.3^{\circ}\right)$ around nadir, respectively. MODIS and VIIRS radiances are limited to the data within a range of $\pm 10^{\circ}$ from nadir, which is larger than $3.3^{\circ}$ to get a stable aggregated value. For each AIRS footprint, all MODIS radiance measurements within a $6.75-\mathrm{km}$ radius of the AIRS pixel center during each SNO were averaged, respectively, to match the spatial grid. The same method was applied to CrIS nadir pixels for VIIRS within a 7-km radius of a CrIS pixel center. Details of the SNO data processing can be found in [25].

\section{WUCD IMPACT ON THE CALIBRATION OF MODIS AND VIIRS TEBS}

The NASA MODIS L1B 5-min products, NASA AIRS L1B products, NASA S-NPP VIIRS level-1 6-min products provided by the NASA Land Science Investigator-led Processing System, and NOAA CrIS SDR products were used for evaluating the impact of WUCD on the MODIS and the VIIRS TEB calibrated radiance. AIRS-MODIS SNO data and CrIS-VIIRS SNO data were collected first. Then, the evaluations were performed after the matching in both spectral bands and spatial FOV.

In the following discussion, the radiance of a MODIS (or VIIRS) band was converted to brightness temperature (BT) using the Planck function based on the central wavelength of the spectral band. For each MODIS (or VIIRS) band and for each AIRS (or CrIS) FOV, the BT difference (delta_BT) was calculated by

$$
\Delta \mathrm{BT}=\mathrm{BT}_{\text {simulated }}-\mathrm{BT}_{\text {aggregated }}
$$

where "BT $\mathrm{B}_{\text {simulated" }}$ is the simulated BT of a MODIS (or VIIRS) band using AIRS (or CrIS) measurements and " $\mathrm{BT}_{\text {aggregated" }}$ is aggregated MODIS (or VIIRS) BT within an AIRS (or CrIS) FOV pixel.

\section{A. WUCD Impact on MODIS Calibration}

For MODIS, we analyzed four WUCD events (2003/209, 2008/236, 2012/286, and 2016/261) collected for four different years throughout the mission. For each WUCD event, all granules from three consecutive days were used in the SNO data collection. Based on the criteria of SNO data selection outlined in Section III-C2, the six footprints around the center of a scan were selected as AIRS nadir pixels. Typically, one SNO AIRS footprint includes about 140 MODIS pixels. In our analysis, only footprints that were covered by more than $70 \%$ MODIS pixels were accepted in AIRS-MODIS SNO data collection. All SNOs from the four WUCD events were combined together in the following data analysis.

Fig. 3 illustrates the distribution of SNO pixels over the MODIS band $31 \mathrm{BT}$ ranges and the $\mathrm{BB}$ temperature ( $T_{-}$bb) ranges. Each aggregation area in Fig. 3(a) is 2-K BT

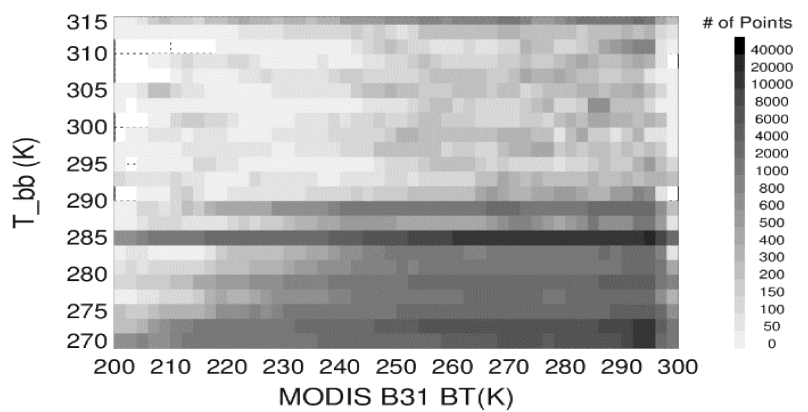

(a)

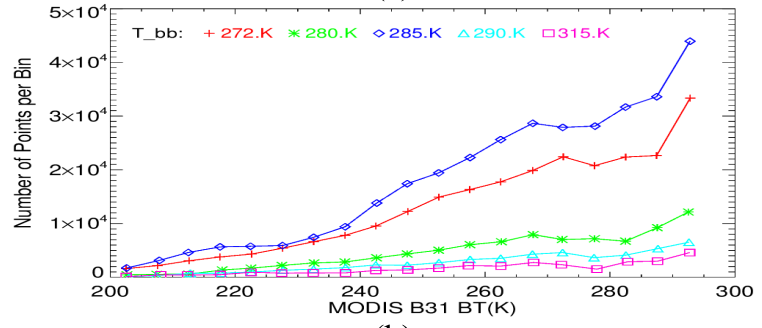

(b)

Fig. 3. AIRS-MODIS SNO FOV distribution as a function of BT and $T \_b b$. (a) Distribution of SNO pixels. (b) Histogram of SNO numbers at a $T \_b$ b.

by 2-K $T \_b b$. During each MODIS WUCD cycle, $T \_b b$ is set to $280,285,290$, and $315 \mathrm{~K}$ for a certain period of time [see Fig. 1(a)], so there are more SNO data when $T \_b b$ is at one of these temperature levels. Since the lowest $T_{-}$bb is larger than $270 \mathrm{~K}$ even though a command sets $T_{-}$bb to $270 \mathrm{~K}$, Fig. 3(b) shows the histogram of SNO numbers at $T_{-}$bb of $272,280,285,290$, and $315 \mathrm{~K}$, respectively. At a given $T \_b b$, more SNOs were collected at the higher MODIS BT range than at lower BT levels, which is earth view scene dependent. Other MODIS bands have similar distributions.

For each aggregation area shown in Fig. 3(a), the differences between AIRS and MODIS measurements from all AIRS footprints within the area were extracted first. Then, the mean difference was determined by the average of the difference data after removing outliers using a 3-sigma filter. Fig. 4 illustrates the distribution of AIRS-MODIS intercomparisons. For the wavelengths of 3.959 and $4.050 \mu \mathrm{m}$ (MODIS bands 22 and 23), the differences between AIRS and MODIS are larger at lower BT. They are not consistent over the range of $T \_$bb for a fixed BT, which indicates that the $T_{-}$bb change impacts the calibration of these two MODIS bands. The distributions of the AIRS-MODIS differences for bands 22 and 23 are similar. For the MODIS bands 31 and 32 with wavelengths at 11 and $12 \mu \mathrm{m}$, larger AIRS-MODIS differences are also observed at lower BT. However, the $T \_$bb change has very limited impact on MODIS bands 31 and 32, which is demonstrated by the consistency of the AIRS-MODIS differences over the $T_{-}$bb range.

To evaluate the impact of the MODIS WUCD cycle on the MODIS L1B products, AIRS-MODIS differences at certain MODIS BT levels were referenced to the operational BB temperature value $(285 \mathrm{~K})$ for all $T_{-}$bb during a MODIS WUCD cycle. The residual is the impact bias. Fig. 5 shows the impact bias over MODIS BT range at $T \_$bb of 272,280 , 


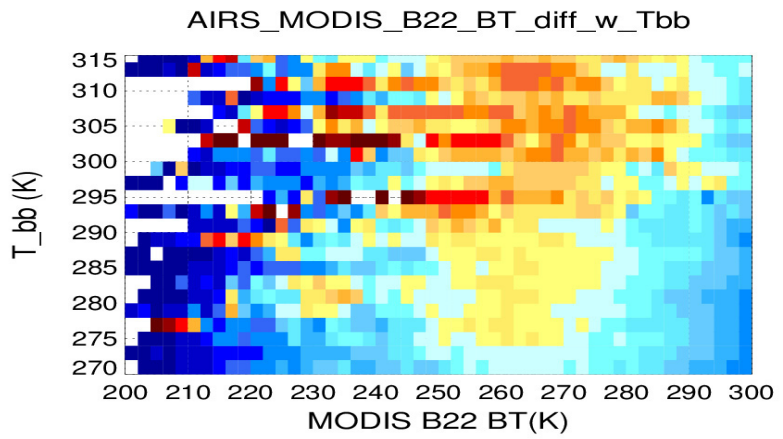

AIRS_MODIS_B23_BT_diff_w_Tbb

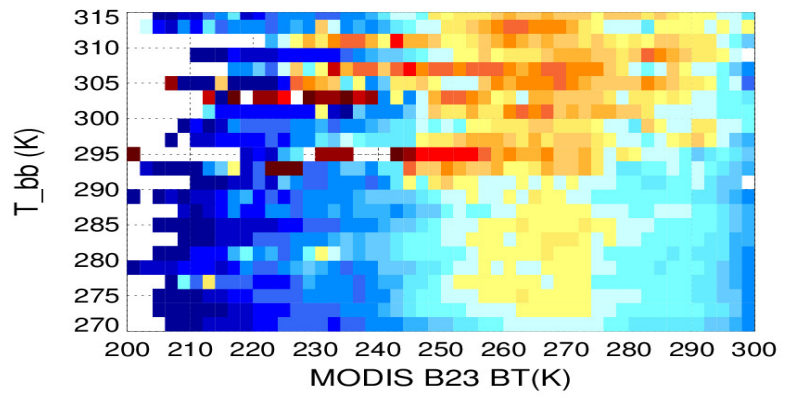

AIRS_MODIS_B31_BT_diff_w_Tbb

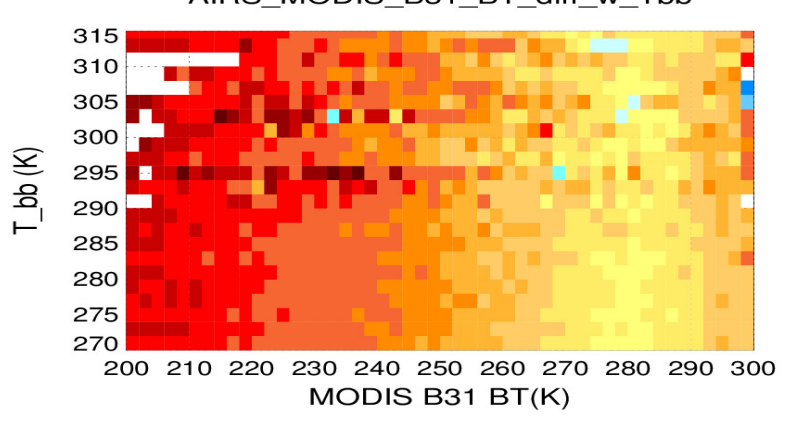

AIRS_MODIS_B32_BT_diff_w_Tbb

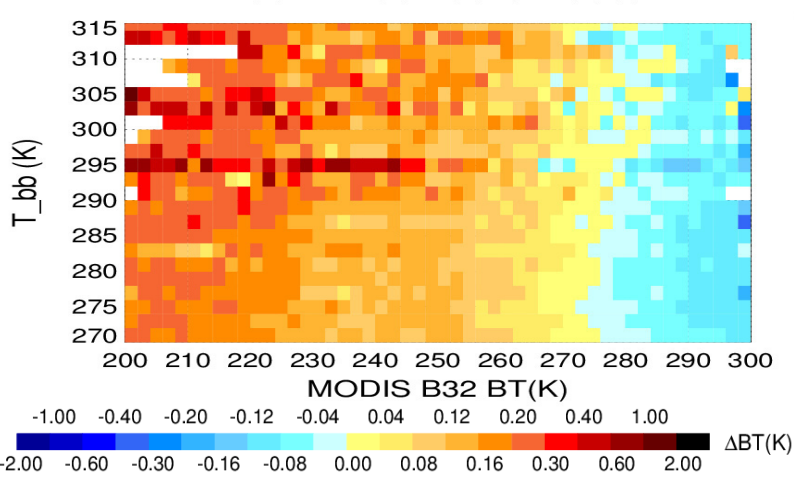

Fig. 4. Distribution of AIRS-MODIS BT difference.

285,290 , and $315 \mathrm{~K}$, where there are more SNO data points. As shown in Fig. 5, more fluctuations are observed when BTs are below $240 \mathrm{~K}$ for all SST bands due to less SNO data. At BT larger than $240 \mathrm{~K}$, MODIS bands 22 and 23 show similar bias, which are less than $0.2 \mathrm{~K}$, while bands 31 and 32 are well below $0.05 \mathrm{~K}$ with some fluctuations at $T_{-}$bb of $315 \mathrm{~K}$.

Since the BB heater is turned ON five times during a MODIS BB warm-up (WU) period, the characterization of TEBs could behave differently from that in the $\mathrm{BB} C D$
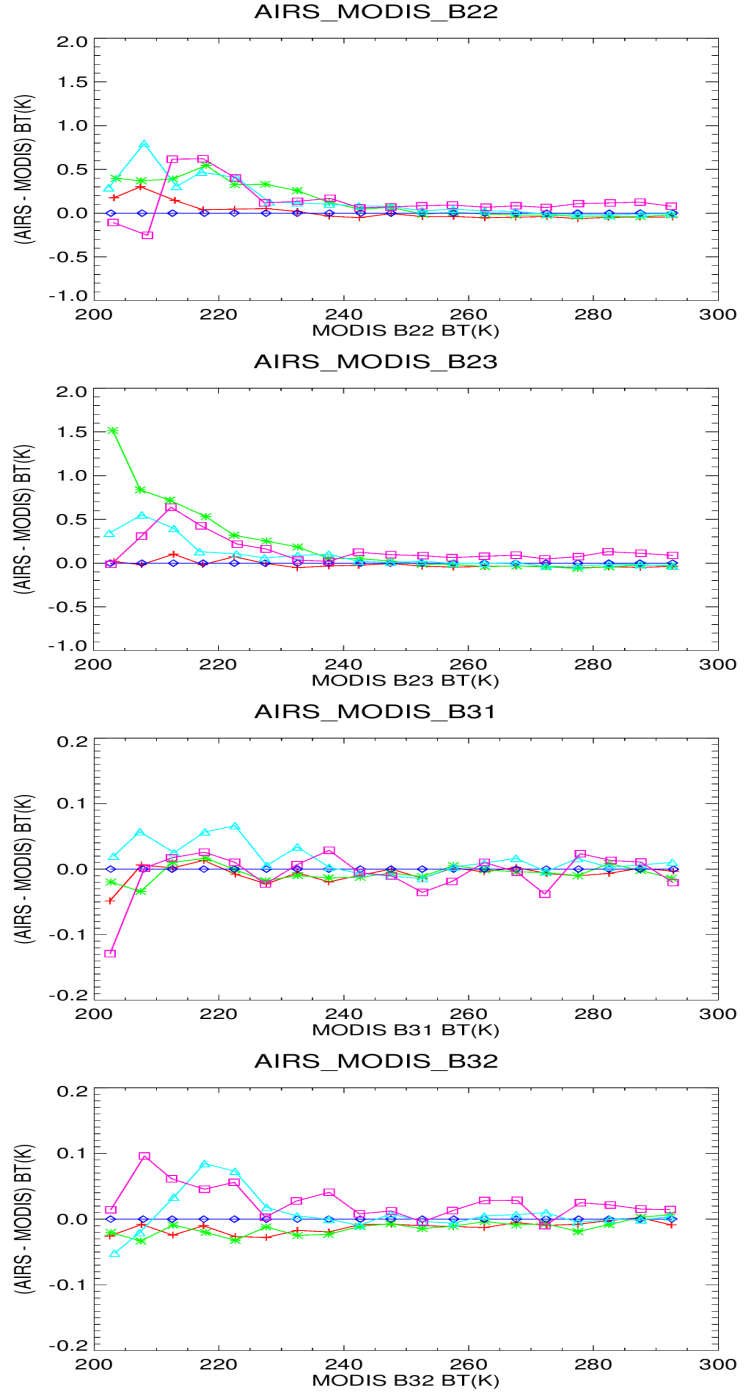

T_bb: $+272 . \mathrm{K} * 280 . \mathrm{K} \diamond 285 . \mathrm{K} \quad \triangle 290 . \mathrm{K} \quad \square 315 . \mathrm{K}$

Fig. 5. Impact on MODIS L1B products during BB WUCD cycle.

period. In order to test this idea, the MODIS granules during warm-up heating (WUH) period and CD period were selected for separate analysis. Any granules during which the $T \_$bb was stable were discarded. Also, the bias between AIRS and MODIS measurements was removed based on the SNO data extracted when MODIS was at its operational $T_{-}$bb value. Fig. 6 shows the $T$ _bb influence on MODIS L1B products during BB WUH and CD periods. The bias is around zero for $T \_$bb less than $290 \mathrm{~K}$. When $T$ bb increases above $290 \mathrm{~K}$, the fluctuations caused by fewer SNO pixels increase and the bias also drifts by about $0.3 \mathrm{~K}$ for bands 22 and 23 and slightly less for bands 31 and 32. As shown in Fig. 6, the pairs of MODIS bands used in SST analysis (bands 22 and 23 for short-wave SST algorithm and bands 31 and 32 for long-wave SST algorithm) show similar features. The fluctuations shown in bands 31 and 32 at $T$ _bb between 290 and $300 \mathrm{~K}$ are caused by 2008/236 and 2012/286 WUCD data, which were impacted by larger fluctuation of cold focal plane assembly tempera- 

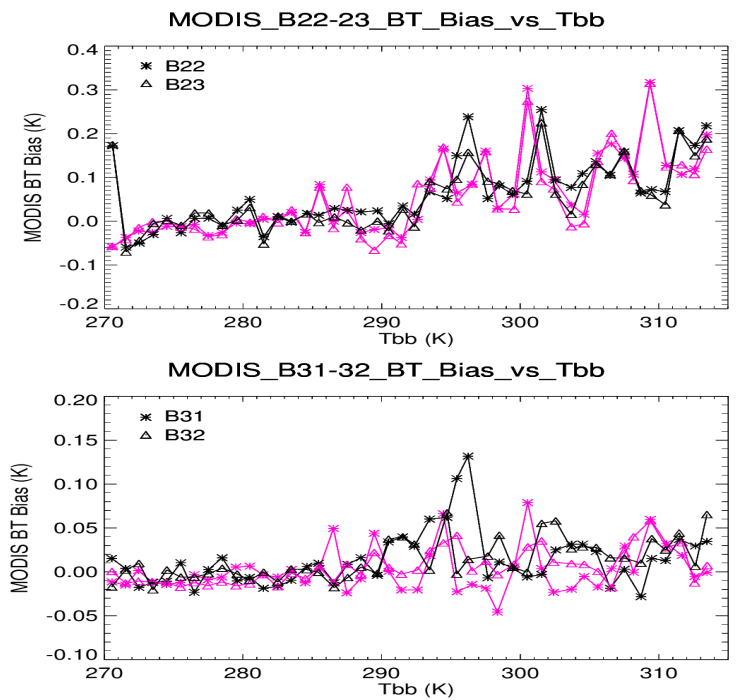

Fig. 6. $\quad T \_b b$ impact on MODIS L1B products during BB WUH (black) and $\mathrm{CD}$ (pink) periods.

ture because of temperature control issue in Aqua MODIS since 2006 [16]. There is no obvious difference between the biases from the WUH and the CD periods.

\section{B. WUCD Impact on VIIRS Calibration}

For evaluating the WUCD impact on VIIRS calibration via CrIS-VIIRS SNO, data from four VIIRS WUCD events (2013/259, 2014/258, 2015/257, and 2016/263) were analyzed. For each WUCD event, $58 \mathrm{~h}$ of granules were used in the SNO data collection, which include $6 \mathrm{~h}$ before and after the WUCD period. According to the SNO data selection criteria outlined in Section III-C2, CrIS SDR nadir pixels were defined as the two center field of regards. Usually, one CrIS FOV contains more than 240 evenly distributed VIIRS pixels (750-m resolution). Only CrIS FOVs that included more than $70 \%$ VIIRS pixels (750-m resolution) were extracted in our SNO data collection to exclude the SNOs with bad or missing VIIRS data. The data analysis results from each of the four WUCD event are very similar. So all SNOs from the four WUCD events were combined together in the following data analysis.

Fig. 7 shows the distribution of SNO pixels over the VIIRS band M15 BT range and its $T \_$bb range. Each square in Fig. 7(a) is $2-\mathrm{K}$ BT by $2-\mathrm{K} T$ _bb. During each VIIRS WUCD cycle, $T$ _bb is set to $297.5,302.5,307.5,312.5$, and $315 \mathrm{~K}$ first. Then, the BB is cooled down from $315 \mathrm{~K}$ to about $266 \mathrm{~K}$. Later on, it is heated to its operational value by setting it to $272.5,282.5$, and $292.5 \mathrm{~K}$ [see Fig. 1(b)]. So there are more SNO data at the $T \_$bb set levels. Similar to MODIS, more SNOs are collected at normal VIIRS BT range than at the extreme BT levels. Other VIIRS bands have

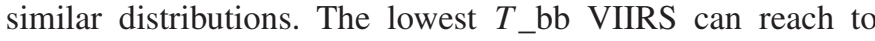
around $266 \mathrm{~K}$, which is lower than MODIS $T \_$bb. Fig. 7(b) shows the histogram of SNOs at $T \_$bb of 272.5, 282.5, 292.5, 297.5, 302.5, 307.5, 312.5, and $315 \mathrm{~K}$.

For each square area in Fig. 7(a), the differences between CrIS and VIIRS measurements from all CrIS footprints within

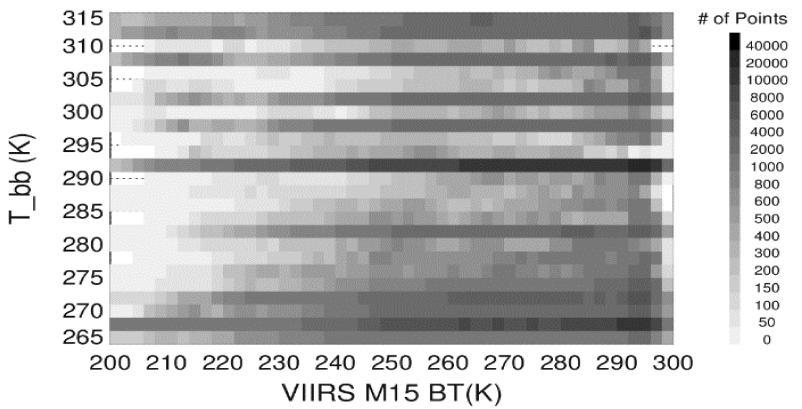

(a)

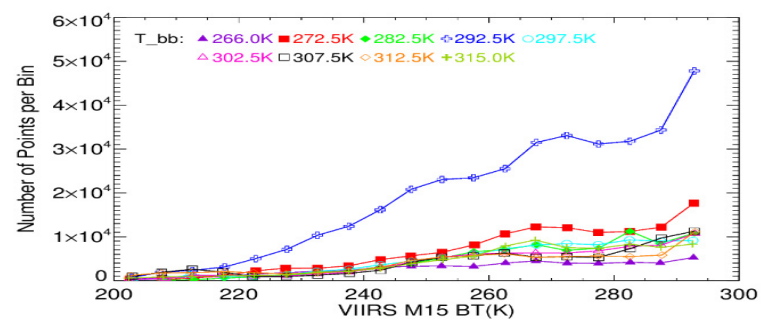

(b)

Fig. 7. CrIS-VIIRS SNO FOV distribution with BT and $T$-bb (a) Distribution of SNO pixels. (b) Histogram of SNO numbers at a $T \_b b$.

that area were averaged after filtering outliers using the same method used in the AIRS-MODIS comparison. Fig. 8 illustrates the distribution of the CrIS-VIIRS intercomparison for all comparable TEBs. The differences between CrIS and VIIRS are mostly within $\pm 0.1 \mathrm{~K}$ and are evenly distributed over the $T$ _bb range for VIIRS bands I5, M13, and M16. Relatively larger differences and uneven distribution are observed in the band M15 comparison, and there are bigger differences at lower BT $(<250 \mathrm{~K})$ than at higher BT.

Similar to the MODIS data analysis, to evaluate the impact of VIIRS WUCD cycle on the VIIRS level-1 products, CrIS-VIIRS differences at a VIIRS BT level were referenced to the operational BB temperature value $(292.5 \mathrm{~K})$ for all $T_{-}$bb values during a VIIRS WUCD cycle. Fig. 9 shows the bias over the VIIRS BT range at $T_{-}$bb of 272.5, 282.5, 292.5, 297.5, 312.5, and $315 \mathrm{~K}$, where $T$ _bb was fixed at these levels for a while so that there are relatively more SNO data points. Less SNOs cause some fluctuations at the lower end of BT. The bias is relatively stable $(<0.05 \mathrm{~K})$ for each $T \_$bb level indicating that the $T_{-}$bb impacts the VIIRS level-1 products.

The TEB behavior during the $\mathrm{BB} \mathrm{WU}$ and $\mathrm{CD}$ periods is also a concern for VIIRS TEB calibration. There are five times when the $\mathrm{BB}$ is heating up at the beginning of a WUCD cycle and three additional times after finishing the CD cycle to recover to the operational $T_{-}$bb level. Similar to MODIS, only the VIIRS granules during WUH and CD periods were selected, excluding any granule for which $T_{-}$bb is constant. The bias between CrIS and VIIRS measurements was removed based on the SNO data extracted at VIIRS operational $T \_$bb value. Fig. 10 illustrates the $T$ _bb influence on VIIRS level-1 products during the BB WUH and CD periods. An obvious, nearly constant, bias between WUH and CD is observed from the plots. In the $T$ _bb range from 265 to $315 \mathrm{~K}$, bands I5, M13, 

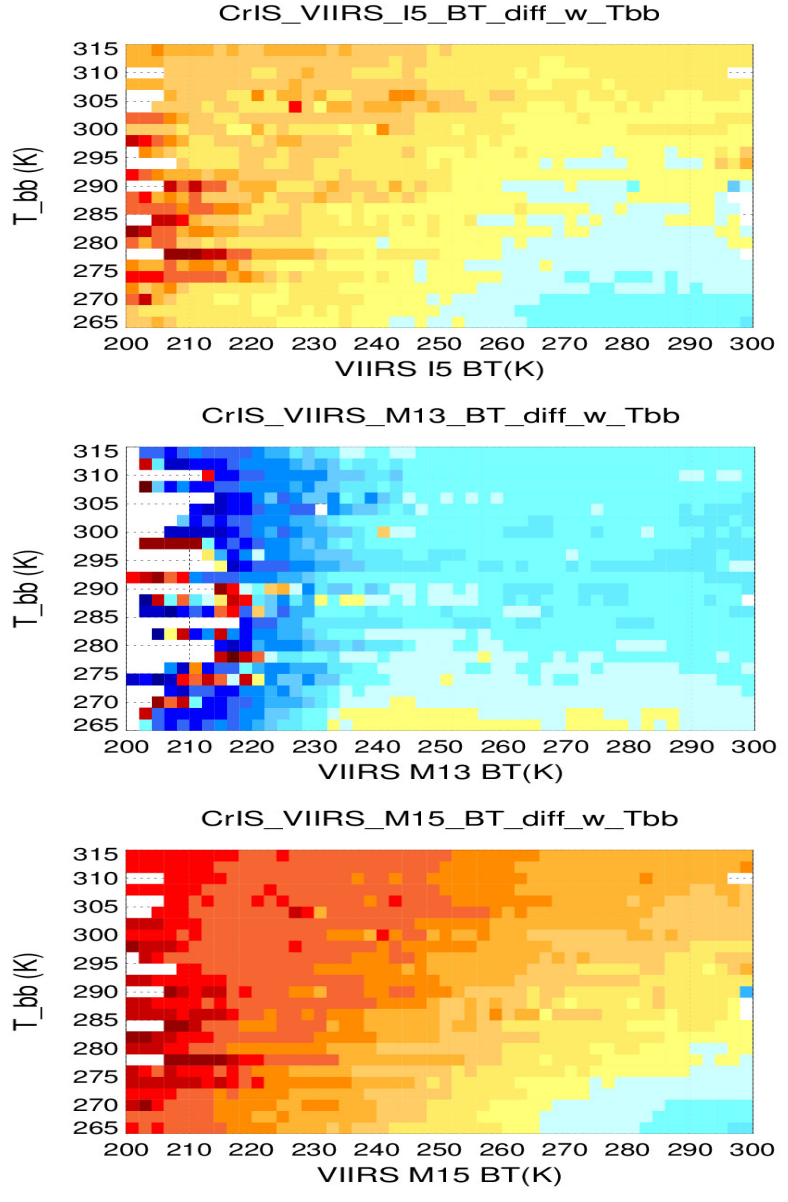

CrIS_VIIRS_M16_BT_diff_w_Tbb

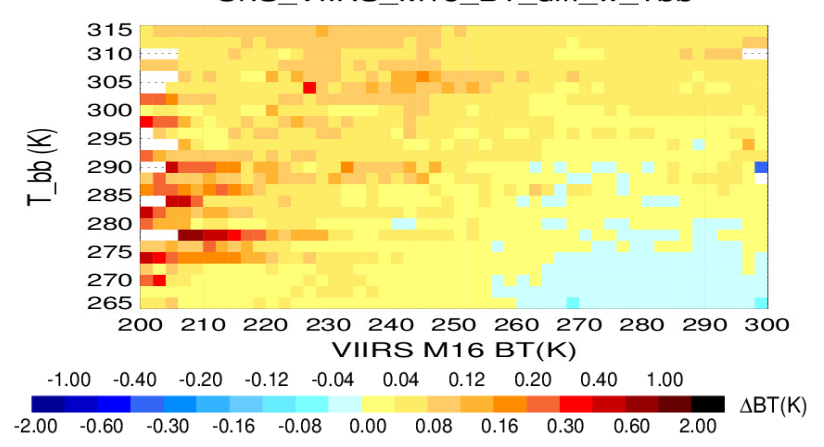

Fig. 8. Distribution of CrIS-VIIRS difference.

and M16 have less than 0.1-K drift while band M15 drifts by around $0.15 \mathrm{~K}$ over the VIIRS BT range. The pair of VIIRS bands used in the long-wave SST algorithm [M15 $(11 \mu \mathrm{m})$ and M16 $(12 \mu \mathrm{m})$ ] shows different trends, which could be the cause of the spikes that appear in SST trending plots during WUCD events.

\section{Discussion}

Figs. 6 and 10 illustrate the $T \_$bb impact on L1B products during $\mathrm{BB}$ WUH and CD periods, respectively, for MODIS and VIIRS. It is observed that different TEBs show different magnitudes of biases when the BB panel experiences temperature changes. The differences between the biases extracted
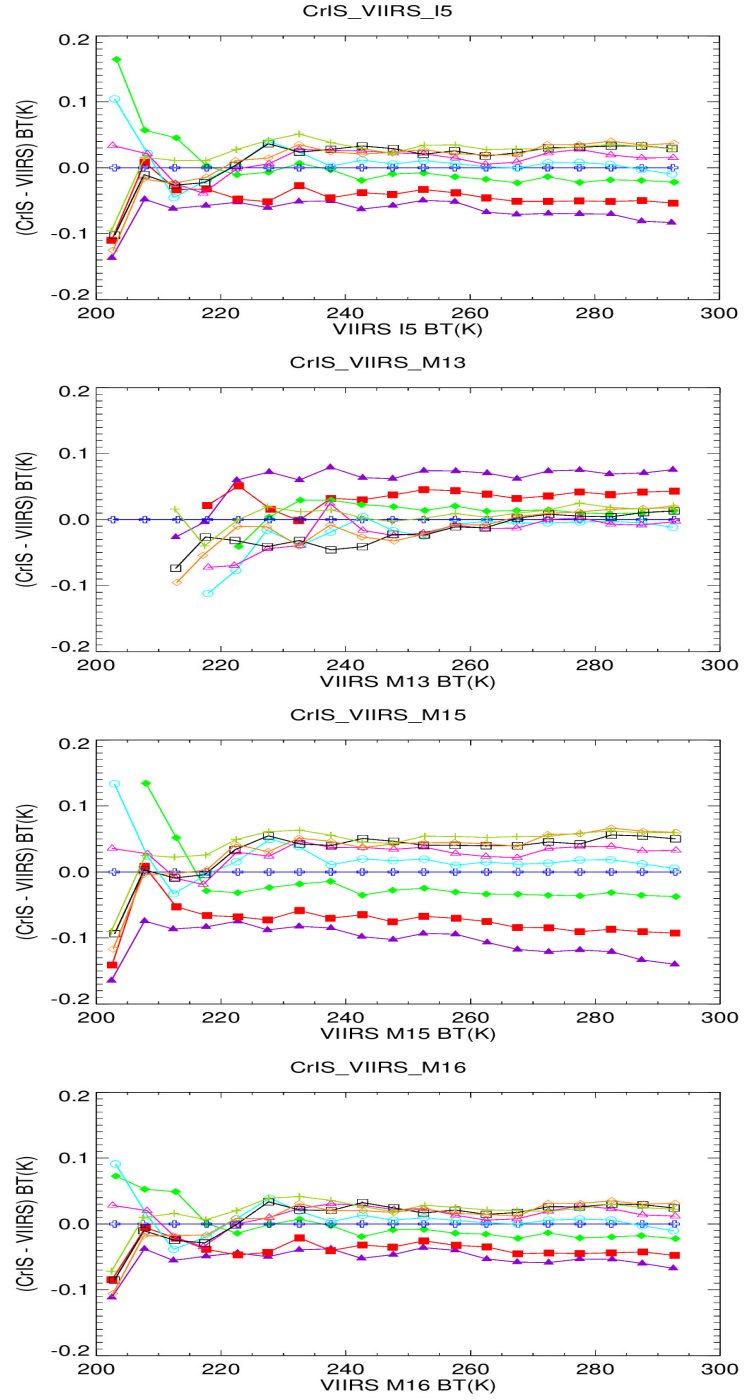

bb: $\Delta 266.0 \mathrm{~K}=272.5 \mathrm{~K} \bullet 282.5 \mathrm{~K}$ ↔292.5K $0297.5 \mathrm{~K} \triangle 302.5 \mathrm{~K} \square 307.5 \mathrm{~K} \diamond 312.5 \mathrm{~K}+315.0 \mathrm{~K}$

Fig. 9. Impact on VIIRS level-1 products during BB WUCD cycle.

from granules in the WUH and CD periods are nearly zero in MODIS bands and are clearly noticeable in VIIRS bands. The reason could be the uniformity of values from individual BB thermistors in the $T$ _bb calculation.

In MODIS, the BB is roughly $8 " \times 14 "$ [27] so that the collected window of each band is well within the angular extent of the BB. The 12 individual $\mathrm{BB}$ thermistors are embedded on the BB panel, two rows of six thermistors evenly spaced along the scan direction, with thermistors \#1 and \#7 on one end of the BB panel and thermistors \#6 and \#12 on the other end [16], [21]. Orbital variation can cause differences among the thermistors. During the BB WU activity, the BB heater on the back of the BB panel is ON in order to control the $\mathrm{BB}$ at a set temperature level. Some components connected with the BB panel (e.g., cable end) may contribute to thermal nonuniformity. Therefore, the heating results in temperature gradients in the BB substrate [21], which produces larger temperature differences among the 12 individual thermistor readings, especially at the higher end of $T_{-}$bb. In contrast, 

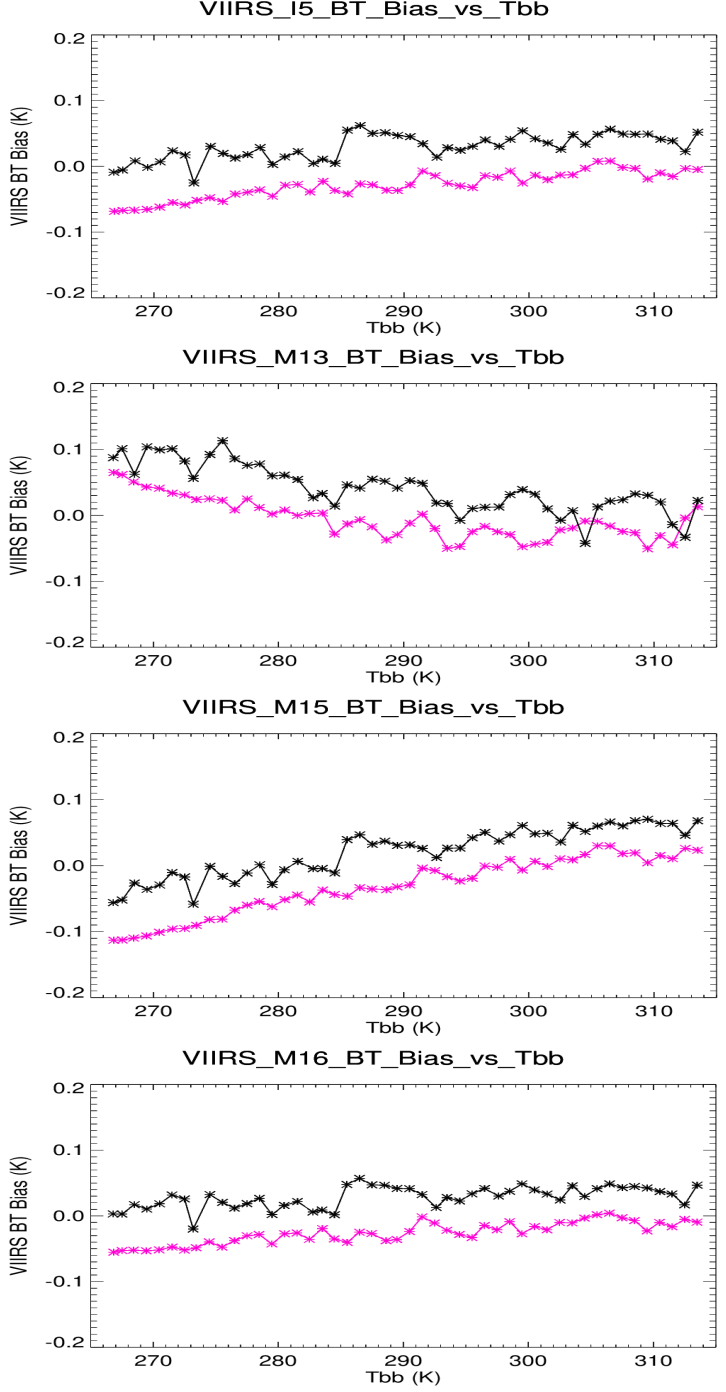

Fig. 10. T_bb impact on VIIRS level-1 products during BB WUH (black) and $\mathrm{CD}$ (pink) periods.

during the $\mathrm{BB} \mathrm{CD}$ period, the temperature of the $\mathrm{BB}$ panel is more uniform because the heater is switched OFF and there is no cooling system in the BB. Fig. 11(a) shows the variation of the 12 individual thermistor readings relative to their average during the September 17, 2016 Aqua MODIS WUCD cycle. Each point in the plot is a granule averaged temperature. Since MODIS BB temperature is determined by the average of the 12 individual $\mathrm{BB}$ thermistor readings after applying a 3-sigma filter, the impact of uneven individual BB thermistor readings is not obvious in the average.

In VIIRS, there is a 2-by-3 array of six active temperature sensors embedded in the BB panel, with thermistors \#1 and \#4 on one end and thermistors \#3 and \#6 on the other end (farthest from the EV port) in the along-scan direction [15], [28]. It has been observed that thermistors \#3 and \#6 are driving the $T \_b b$ variation likely due to earth illumination heating one side of the BB [15]. Like MODIS, the BB CD operation is also a passive process. Fig. 11(b) illustrates the variation of the six $\mathrm{BB}$ thermistor readings relative to their average during the September 19, 2016 VIIRS WUCD cycle. As shown in

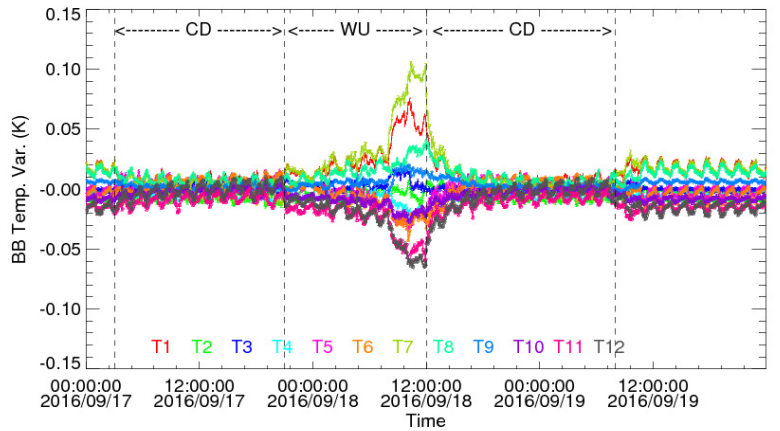

(a)

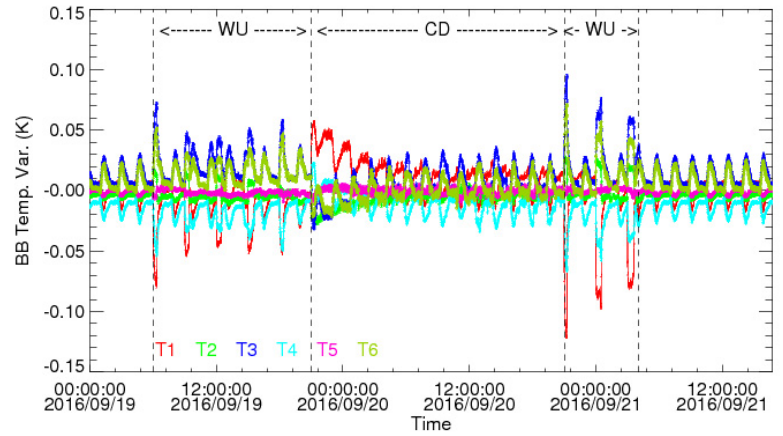

(b)

Fig. 11. Variations of individual BB thermistor readings relative to their average during a WUCD cycle. (a) MODIS September 17, 2016. (b) VIIRS September 19, 2016.

the plot, the distribution of the relative differences between individual thermistors and their average is not consistent during WU and CD periods, which means that the contribution of each thermistor reading acts differently in the calculation of the averaged $T \_$bb. In VIIRS TEB calibration, the readings from all six BB thermistors are averaged with equal weights, which is acceptable during the normal operations [12].

In the current MODIS Collection 6 TEB calibration algorithm, the calibration coefficients $a_{0}$ and $a_{2}$ are estimated using the data during $\mathrm{BB} C \mathrm{CD}$ period so as to have a better BB uniformity. Although the current VIIRS TEB calibration algorithm is based on prelaunch data, its BB CD data are still used for monitoring calibration coefficients. Reanalysis of prelaunch data may bring some information for TEB calibration algorithm improvements, such as whether all factors that impact the calibration quality are characterized accurately and the $\mathrm{BB}$ panel nonuniformity characterization in the $\mathrm{BB}$ temperature determination [21]

BB WUCD impact has been observed in the SST time series of the 24-h statistics over global ocean clear-sky pixels [17]. The SST is determined using data from 11- and $12-\mu \mathrm{m}$ bands. For example, SST is calculated from MODIS data by

$$
\begin{aligned}
& \mathrm{SST}_{\text {modis }}=k_{1}+k_{2} \cdot \mathrm{BT}_{31}+k_{3} \cdot \mathrm{BT}_{3132}+k_{4} \\
& \cdot[\sec (\theta)-1] \cdot \mathrm{BT}_{3132}
\end{aligned}
$$

where $k_{i}(i=1,2,3,4)$ are coefficients for SST retrieval, $\mathrm{BT}_{31}$ is the band $31 \mathrm{BT}, \mathrm{BT}_{3132}=\mathrm{BT}_{32}-\mathrm{BT}_{31}$, and $\theta$ is the satellite zenith angle [4]. A similar approach is implemented for VIIRS SST extraction using bands M15 and M16 [6]. 
From Fig. 6, we can see that MODIS BB WUCD have similar impact on bands 31 and 32 with less than $0.05-\mathrm{K}$ BT bias fluctuations on average during the entire WUCD cycle. However, there is about $0.15 \mathrm{~K}$ total impact to VIIRS M15 $(11 \mu \mathrm{m})$, as seen in Fig. 10. In addition, the behavior of M15 is different from that of M16 (12 $\mu \mathrm{m})$, which adds another bias based on the SST formula. This could be the reason that spikes were observed during WUCD events in VIIRS SST trending but were not seen in the MODIS SST time series.

The results of four VIIRS TEBs (I5, M13, M15, and M16) spectrally overlapping with CrIS are presented in this paper. In addition to the MODIS bands used in the SST application, all other MODIS TEBs (B21, 24 and 25, 27 and 28, 30, and 33-36) overlapped with AIRS in spectra were also evaluated. For the MODIS bands with saturation issues (bands 33, 35, and 36 ), there are less than $0.1-\mathrm{K}$ bias when the bands are not saturated and $0.2-0.4 \mathrm{~K}$ bias when they are saturated. For the other bands, the maximum biases range from $\sim 0.1 \mathrm{~K}$ (bands 25,28 , and 30 ) to a maximum of $0.4-0.5 \mathrm{~K}$ (bands 21, 24, and 27) with no obvious trend versus $T \_b b$.

To mitigate the impact of BB WUCD, Cao et al. [26] have proposed a compensatory term in the TEB calibration equation to try to find the difference of calibration profile between prelaunch and postlaunch. Based on our study, the difference actually arises from the variations of individual $\mathrm{BB}$ thermistor readings. We are experimenting with different methods to reduce the impact of the BB WUCD operation on the TEB calibration, e.g., properly weighting the individual $\mathrm{BB}$ thermistor readings in their average by including the characterization of the BB panel nonuniformity. This improvement will be further investigated in our future work.

\section{SUMMARY}

This paper evaluates the calibrated data for the TEBs from Aqua MODIS and S-NPP VIIRS during their BB WUCD cycles, mainly focusing on the bands used in the SST products. The evaluation approach is via the SNOs with the reference measurements from hyperspectral sounders (AIRS and CrIS). The results show that for MODIS bands 22 and 23, and 31 and 32, the impact is around zero for BB temperature below $290 \mathrm{~K}$ and up to $0.3 \mathrm{~K}$ for bands 22 and 23 and $0.05 \mathrm{~K}$ for bands 31 and 32, respectively, when the $\mathrm{BB}$ temperature is beyond $290 \mathrm{~K}$. For S-NPP VIIRS, drifts observed in M15 and M16 are likely dependent on the BB temperature. The overall drifts from the lowest $T \_b b(266 \mathrm{~K})$ to the highest $T$ bb $(315 \mathrm{~K})$ can be 0.15 and $0.1 \mathrm{~K}$ for M15 and M16, respectively, which result in a change in the derived SST.

\section{REFERENCES}

[1] C. Cao, F. J. De Luccia, X. Xiong, R. Wolfe, and F. Weng, "Early on-orbit performance of the visible infrared imaging radiometer suite onboard the Suomi National Polar-Orbiting Partnership (S-NPP) satellite," IEEE Trans. Geosci. Remote Sens., vol. 52, no. 2, pp. 1142-1156, Feb. 2014.

[2] X. Xiong et al., "VIIRS on-orbit calibration methodology and performance," J. Geophys. Res. Atmos., vol. 119, no. 9, pp. 5065-5078, May 2014.
[3] Z. Wan and Z.-L. Li, "A physics-based algorithm for retrieving landsurface emissivity and temperature from EOS/MODIS data," IEEE Trans. Geosci. Remote Sens., vol. 35, no. 4, pp. 980-996, Jul. 1997.

[4] O. B. Brown et al., "MODIS infrared sea surface temperature algorithm-Algorithm theoretical basis document version 2.0," Univ. Miami, Apr. 1999. [Online]. Available: https://modis.gsfc.nasa.gov/data/atbd/atbd_mod25.pdf

[5] Joint Polar Satellite System (JPSS). (2013). VIIRS Land Surface Temperature Algorithm Theoretical Basis Document (ATBD), Goddard Space Flight Center, Greenbelt, MD, USA. [Online]. Available: https://www. star.nesdis.noaa.gov/jpss/documents/ATBD/D0001-M01-S01-022_JPSS _ATBD_VIIRS-LST_A.pdf

[6] Joint Polar Satellite System (JPSS). VIIRS Sea Surface Temperature Algorithm Theoretical Basis Document (ATBD) Revision A, Goddard Space Flight Center, Greenbelt, MD, USA. [Online]. Available: https://www.star.nesdis.noaa.gov/jpss/documents/ATBD/D0001-M01S01-010_JPSS_ATBD_VIIRS-SST_A.pdf

[7] S. A. Ackerman, K. I. Strabala, W. P. Menzel, R. A. Frey, C. C. Moeller, and L. E. Gumley, "Discriminating clear sky from clouds with MODIS," J. Geophys. Res., vol. 103, no. D24, pp. 32141-32157, Dec. 1998.

[8] X. Xiong, K. F. Chiang, A. Wu, W. L. Barnes, B. Guenther, and V. V. Salomonson, "Multiyear on-orbit calibration and performance of terra MODIS thermal emissive bands," IEEE Trans. Geosci. Remote Sens., vol. 46, no. 6, pp. 1790-1803, Jun. 2008.

[9] X. Xiong, B. N. Wenny, A. Wu, W. L. Barnes, and V. V. Salomonson, "Aqua MODIS thermal emissive band on-orbit calibration, characterization, and performance," IEEE Trans. Geosci. Remote Sens., vol. 47, no. 3, pp. 803-814, Mar. 2009.

[10] C. Moeller, D. Tobin, and G. Quinn, "S-NPP VIIRS thermal band spectral radiance performance through 18 months of operation on-orbit," Proc. SPIE, vol. 8866, Sep. 2013, Art. no. 88661N.

[11] C. Cao et al., "Suomi NPP VIIRS sensor data record verification, validation, and long-term performance monitoring," J. Geophys. Res. Atmos., vol. 118, no. 20, pp. 11664-11678, Oct. 2013.

[12] B. Efremova, J. McIntire, D. Moyer, X. Xiong, and A. Wu, "S-NPP VIIRS thermal emissive bands on-orbit calibration and performance," J. Geophys. Res. Atmos., vol. 119, no. 18, pp. 10859-10875, Sep. 2014.

[13] D. C. Tobin, H. E. Revercomb, C. C. Moeller, and T. S. Pagano, "Use of atmospheric infrared sounder high-spectral resolution spectra to assess the calibration of Moderate resolution Imaging Spectroradiometer on EOS Aqua," J. Geophys. Res., vol. 111, no. D9, May 2006, Art. no. D09S05.

[14] B. N. Wenny and X. Xiong, "Using a cold Earth surface target to characterize long-term stability of the MODIS thermal emissive bands," IEEE Geosci. Remote Sens. Lett., vol. 5, no. 2, pp. 162-165, Apr. 2008.

[15] X. Xiong et al., "Assessment of S-NPP VIIRS on-orbit radiometric calibration and performance," Remote Sens., vol. 8, no. 2, Jan. 2016, Art. no. 84.

[16] B. N. Wenny et al., "MODIS TEB calibration approach in collection 6," Proc. SPIE, vol. 8533, Nov. 2012, Art. no. 85331M.

[17] T. J. Choi, C. Cao, and F. Weng, "S-NPP VIIRS thermal emissive band gain correction during the blackbody warm-up-cool-down cycle," Proc. SPIE, vol. 9972, Sep. 2016, Art. no. 99721J.

[18] D. A. Elliott and H. H. Aumann, "Comparison of AIRS and IASI surface observations of DomeC in antarctica with surface temperatures reported by AWS8989," in Proc. AGU Fall Meeting, Dec. 2008.

[19] L. Wang et al., "Consistency assessment of Atmospheric Infrared Sounder and Infrared Atmospheric Sounding Interferometer radiances: Double differences versus simultaneous nadir overpasses," J. Geophys. Res., vol. 116, no. D11, Jun. 2011, paper D11203.

[20] D. Tobin et al., "Suomi NPP/JPSS cross-track infrared sounder (CRIS): Intercalibration with AIRS, IASI, and VIIRS," in Proc. AMS Аnnu. Meeting, Austin, TX, USA, Jan. 2013, p. P301.

[21] X. Xiong, B. N. Wenny, A. Wu, and W. L. Barnes, "MODIS onboard blackbody function and performance," IEEE Trans. Geosci. Remote Sens., vol. 47, no. 12, pp. 4210-4222, Dec. 2009.

[22] T. Chang and X. Xiong, "Assessment of MODIS thermal emissive band on-orbit calibration," IEEE Trans. Geosci. Remote Sens., vol. 49, no. 6, pp. 2415-2425, Jun. 2011.

[23] A. Wu et al., "Adjusting Aqua MODIS TEB nonlinear calibration coefficients using iterative solution," Proc. SPIE, vol. 9264, Dec. 2014, Art. no. 92640R 
[24] Joint Polar Satellite System (JPSS). (2013). VIIRS Radiometric Calibration Algorithm Theoretical Basis document (ATBD), Revision C, Goddard Space Flight Center, Greenbelt, MD, USA. [Online]. Available: https://www.star.nesdis.noaa.gov/jpss/documents/ATBD/D0001-M01S01-003_JPSS_ATBD_VIIRS-DR_C.pdf

[25] Y. Li, A. Wu, and X. Xiong, "Inter-comparison of S-NPP VIIRS and Aqua MODIS thermal emissive bands using hyperspectral infrared sounder measurements as a transfer reference," Remote Sens., vol. 8, no. 1, Jan. 2016, Art. no. 72.

[26] C. Cao, W. Wang, S. Blonski, and B. Zhang, "Radiometric traceability diagnosis and bias correction for the Suomi NPP VIIRS long-wave infrared channels during blackbody unsteady states," J. Geophys. Res. Atmos., vol. 122, no. 10, pp. 5285-5297, May 2017.

[27] X. Xiong, N. Che, S. Xiong, K. Chiang, and W. Barnes, "Performance of the Terra MODIS on-board blackbody," Proc. SPIE, vol. 5882, Aug. 2005, Art. no. 58820U

[28] X. Xiong et al., "Comparison of MODIS and VIIRS onboard blackbody performance," Proc. SPIE, vol. 8533, Nov. 2012, Art. no. 853318.

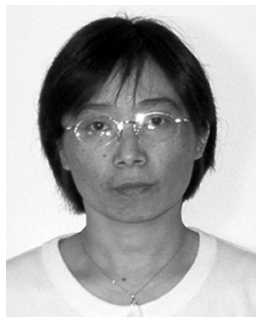

Yonghong Li (M'18) received the Ph.D. degree in electrical engineering from the University of Missouri, Columbia, MO, USA.

She was involved in remote sensing image processing, ice sheet change detection, and marine radar signal processing. She is currently with both Moderate Resolution Imaging Spectroradiometer and Visible Infrared Imaging Radiometer Suite Characterization and Support Teams on the radiometric characterization and calibration of remote sensing instruments.

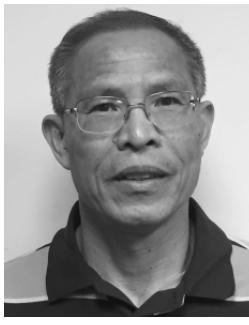

Xiaoxiong (Jack) Xiong received the B.S. degree in optical engineering from the Beijing Institute of Technology, Beijing, China, and the Ph.D. degree in physics from the University of Maryland, College Park, MD, USA.

He was involved in optical instrumentation, nonlinear optics, laser and atomic spectroscopy, and resonance ionization mass spectrometry at universities, industry, and the National Institute of Standards and Technology. He is an Optical Physicist with the Goddard Space Flight Center, National Aeronautics and Space Administration, Greenbelt, MD, USA, where he is also the Moderate Resolution Imaging Spectroradiometer (MODIS) Project Scientist and the Technical Lead for both the MODIS Characterization Support Team and the Visible Infrared Imaging Radiometer Suite Characterization Support Team.

Jeff McIntire, photograph and biography not available at the time of publication.

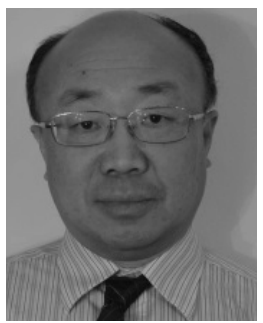

Aisheng Wu received the B.S. degree in atmospheric science from the University of Science and Technology of China, Hefei, China, the M.Sc. degree in atmospheric remote sensing from the Institute of Plateau Atmospheric Physics, Chinese Academy of Science, Lanzhou, China, and the Ph.D. degree in biometeorology/soil physics from The University of British Columbia, Vancouver, BC, Canada.

$\mathrm{He}$ is currently with both Moderate Resolution Imaging Spectroradiometer and Visible Infrared Imaging Radiometer Suite Characterization and Support Teams, Goddard Space Flight Center, National Aeronautics and Space Administration, Greenbelt, MD, USA. 